\title{
Typing of Four Cases of Monoclonal Gammopathy: A Revival of Immunosubstraction Role
}

\author{
Marta Monari ${ }^{1}$, Paolo Bianchi ${ }^{1}$, Federica Maura' ${ }^{1}$, Lucia Motta ${ }^{1}$, Giovanni Martellasio², \\ Francesco Paolo Leone ${ }^{1}$, Alessandro Montanelli ${ }^{2}$
}

${ }^{1}$ Clinical Investigation Laboratory, Humanitas Clinical and Research Hospital, Rozzano, Milano

${ }^{2}$ Laboratorio Analisi Cliniche, Spedali Civili di Brescia, Brescia, Italy

Email: marta_noemi.monari@humanitas.it

How to cite this paper: Monari, M., Bianchi, P., Maura, F., Motta, L., Martellasio, G., Leone, F.P. and Montanelli, A. (2017) Typing of Four Cases of Monoclonal Gammopathy: A Revival of Immunosubstraction Role. Health, 9, 1852-1857. https://doi.org/10.4236/health.2017.913134

Received: July 5, 2017

Accepted: December 26, 2017

Published: December 29, 2017

Copyright () 2017 by authors and Scientific Research Publishing Inc. This work is licensed under the Creative Commons Attribution International License (CC BY 4.0).

http://creativecommons.org/licenses/by/4.0/

\begin{abstract}
Monoclonal gammopathy of undetermined significance (MGUS) is characterized by increased production of an immunoglobuling (Ig) from a clone of plasma cells and is a pre-malignant disorders in subjects older than 50 years. The prevalence of MGUS in Caucasian population is still not determined. MGUS is characterized by the presence of a monoclonal-protein(M-protein) (IgG and IgA) lower than $30 \mathrm{~g} / \mathrm{L}$, bone marrow plasma cell percentage lower than $10 \%$, and absence of clinical signs related to multiple myeloma (MM). MGUS can be responsible for damage to organs through the production of toxic M proteins that may have autoantibody activity or deposit pathologically in the organ tissues. Many techniques are available for the characterization of M-proteins. These techniques can involve different expenses, skills, labor time, and sensitivity in detecting monoclonal proteins also at low-level. Detection of M-proteins needs of assays based on high-resolution electrophoresis and immunofixation (or immunosubtraction). We show suggestive clinical cases where the subjects involved had not an apparent disease but they showed an interesting pattern in electrophoresis. All cases were investigated by capillary's electrophoresis and immunofixation to confirm or not the clinical suspect, and then if the immunofixation is not exhaustive, additionally immunosubstraction is done. However in some cases, the interpretation of the peaks is not so easy. Clinical and scientific data provided evidences that immunofixaction technique can fail the identification of monoclonal components. In that cases, we opted for the immunosubtraction method as a third level test, in that cases when immunofixation failed the identification of a monoclonal protein.
\end{abstract}




\section{Keywords}

MGUS-Monoclona Gammopathy of Undetermined Significance, Immunofixation, Immunosubstraction, Capillary's Electrophoresis

\section{Introduction}

An increased production or an overproduction of an immunoglobulin (Ig), derived from a clone of plasma cells is a clinical feature of monoclonal gammopathies. Immunoglobulin overproduction is often detectable in subjects with pre-malignant disease, who have no sign or symptoms that can be associated to monoclonal protein, and this clinical condition is called monoclonal gammophaty of undetermined significance (MGUS). This pre-malignant condition is quite common in subjects' alder than 50 years [1] [2]. In a recent study aimed to investigate the prevalence of pre-malignant plasma-cell disorders among subjects of 50 years of age or older, it has been reported that the prevalence of MGUS was 3.2\%. The age adjusted rates of MGUS were higher in men than in women ( $4.0 \%$ vs. $2.7 \%$ respectively). In addition in subjects older than 70 years the prevalence of MGUS was 5.3 and reached $7.5 \%$ in people older than 80 years [2]. MGUS show a higher prevalence in blacks than in whites subjects [3]. The annual incidence of MGUS in the European Caucasian population remains unclear: a study by the Haematological Malignancy Research Network (HMRN) showed an annual incidence rate of 6.2 per 100,000 subjects, in the UK (20042008) [4]. This rate was pretty close to the $M M$ in the same study (6.1 per 100,000). It has been shown that WSR for MGUS was $3.76 \pm 0.26$ per 100,000 inhabitants, increasing regularly with age. We also observed a rate of progression to MM of $1.41 \%$ per year, a rate identical to that of the Mayo Clinic (1.5\%) [5] [6]. MGUS is characterized by a content of M-protein $\leq 30 \mathrm{~g} / \mathrm{L}$, with a percentage of bone marrow plasma cell $<10 \%$ and no clinical signs of multiple myeloma (MM) [2] [7]. MGUS can be also responsible of severe organ damage through the toxic M-proteins production that can have autoantibody activity or to deposit him in pathological way in the organ tissues. Recently has been shown that microenvironment precancerous modifications induced by M-proteins might increase the risk of venous and arterial thrombosis, infections and the osteoporosis [8]. At today there are no clear data about risk factors for MGUS, and about possible genetic factors associate to the MGUS [9] [10]. Noteworthy, in acquired immunodeficiency caused by drug treatments after organ transplantations can appear a monoclonal gammopathy that can disappears when the normal immune system role is restored [11], but much rarer that a disappearance of a monoclonal protein may occur without a therapeutic intervention [9].

A feature of monoclonal gammopathies is the rearrangement of immunoglobulins genes resulting in the overproduction of a monoclonal protein [9] [10] [11]. 
The diagnosis of MGUS is usually detected as a homogenous electrophoretic peak in serum protein and results as an incidental finding during routine blood tests. In this patient generally there are no clinical signs related to this monoclonal component. Patients with MGUS fulfilling the criteria for Multiple Myeloma with no symptoms or clinical signs are categorized as patients with smoldering multiple myeloma [12]. Many studies investigated both the rate of progression and predictive markers associated to the malignant progression in patients with MGUS, and the hazard rate was similar in all the studies with a long time follow up [13], showing a likelihood of malignant progression at 20 years comprised from $12 \%$ to $17 \%$. The greatest chance of progression was estimated in younger subjects, thus making the rate of progression not influenced by age [3].

MGUS can also cause autoimmune diseases, due to the autoantibody properties of M-protein when they are accumulated in tissue organs. Moreover, it was reported that in bone marrow microenvironment, pre-malignat clone can cause alterations resulting in an increased risk of thrombosis (venous and arterial), infections and osteoporosis [8].

In this study, we present four suggestive clinical cases where the subjects involved had not an apparent disease but they showed a very interesting pattern in electrophoresis.

\section{Methods}

In our laboratory all patients of National Health Service that showed an unknown serum monoclonal suspected component capillary's electrophoresis were investigated by immunofixation to confirm or not the suspect. If the immunofixation is not exhaustive, additionally immunosubstraction is done.

We received, in 6 months, 4086 examination requirements for electrophoresis and for 450 (11\%) we have done the confirmation of monoclonal suspected gammopathy. At the first step, we use Capillarys 2 electrophoresis by Sebia ${ }^{\circ}$ (sensitivity $04 \%$, specificity $99 \%$ ); for eventually confirmation Immunofixation on IF-Hydrasys Sebia ${ }^{\circledR}$ with common anti-serum anti IgG-IgA, IgM, Kappa and Lambda (sensitivity 91\%, specificity $98 \%$ ). In some cases, where the precedent anti-serum were non exhaustive, we proceed with $\operatorname{IgD}$, IgE kappa and Lambda. In addition for the serum that are not resolutive with the gel we continue with Sebia Capillarys $2.0^{\circ}$ for immunosubtraction (sensitivity $95 \%$, specificity $99 \%$ ).

\section{Results}

All 450 immunofix have shown a monoclonal component, in four cases we observed uncommon pattern of fixation: (Table 1)

Case 1: In gel IgM-K and IgA not related to $\mathrm{K}$ or $\lambda \mathrm{f}$-chains.

Case 2: In gel IgG-K, IgA and $\lambda$ free at different height on gel.

Case 3: In gel IgG-K and IgA not related to $\mathrm{K}$ or $\lambda$ free.

Case 4: In gel IgG-K and IgA not related to $\mathrm{K}$ or $\lambda$ chains. 
Table 1. Laboraty findings of the four interesting cases by immunofix using three different laboratory methods.

\begin{tabular}{|c|c|c|c|}
\hline & Capillarys & Gel fix & Immunotyping \\
\hline Case 1 & $\begin{array}{l}\text { Only one suspected } \\
\text { component in gamma }\end{array}$ & $\begin{array}{c}\operatorname{IgM}-\mathrm{K} \\
\text { IgA not related to } \mathrm{K} \text { or } \lambda\end{array}$ & $\operatorname{IgM}-\mathrm{K}$ and $\operatorname{Ig} \mathrm{A}-\lambda$ \\
\hline Case 2 & $\begin{array}{l}\text { Bridge between B2 and } \\
\text { gammaglobulins and suspected } \\
\text { component in gammaglobulins }\end{array}$ & $\begin{array}{c}\text { IgG-K } \\
\text { IgA and } \lambda \text { chains } \\
\text { on different height }\end{array}$ & IgG-K and $\lambda$ chains \\
\hline Case 3 & $\begin{array}{l}\text { suspected component } \\
\text { in gammaglobulins }\end{array}$ & $\begin{array}{c}\operatorname{IgG}-\mathrm{K} \\
\text { IgA not related to } \mathrm{K} \text { or } \lambda\end{array}$ & IgG-K \\
\hline Case 4 & $\begin{array}{l}\text { Bridge between B2 } \\
\text { and gammaglobulins }\end{array}$ & $\begin{array}{c}\text { IgG-K } \\
\text { IgA not related to } \mathrm{K} \text { or } \lambda\end{array}$ & IgG-K \\
\hline
\end{tabular}

For all them we have done the immunotyping and we obtained:

Case 1: IgM-K and IgA- $\lambda$.

Case 2: $\operatorname{IgG}-\mathrm{K}$ and $\lambda$ chains.

Case 3: IgG-K.

Case 4: IgG-k.

In all of them we could underline that the heavy chains component associated to light chain $\mathrm{K}$ was always confirmed and the real troubleshooting are related to the presence of IgA or $\lambda$. I Fifty \% of these cases are not confirmed with a different methods. We also investigated the clinical and laboratory history of the 4 cases and we didn't find any interesting aspect to be associated with these monoclonal components.

\section{Discussion}

Capillary'S electrophoresis is a sensitive, rapid and well-standardization primary screening for the study of proteins patterns. However in some cases it is not so easy the intepretations of the peaks. In this study we have used two different methods: immunofix and in some cases immunosubstraction to investigated the suspected monoclonal gammopaty detectedin the screening test by capillarys electrophoresys: immunofix is conducted on commercial gels of polyacrylamide and used on a first step commercial anti-antibodies $\operatorname{IgG}, \operatorname{IgA}, \operatorname{IgM} \mathrm{K}$ and $\lambda$ chains in additionally it could be used IgD and IgE to confirm or not the presence of light chain; the second one is a capillary's typing used the same antiserum of immunofix but it pull out the presence of monoclonal gammopaty for immunosubtraction of peaks. The combined use of the two methods has been performed to obtain a better resolution of the observations and to give a correct diagnostic and clinical information to patients and to physicians. Diagnosis and management of patients with monoclonal gammopathies depend on the accurate identification and characterization of proteins; multiple myeloma is determined by the presence of clonal expression of malignant in bone marrow cells engaged in the production of a monoclonal immunoglobulin. Occasionally monoclonal pattern, bi-clonal or tri-clonal para-proteins are present at the diagnosis and also 
switching of para-protein can occur during disease relapse [14]. In all of our cases none of the patients has a known malignancies or inflammatory disease. IFE was probably considered the "gold standard" for the detection of monoclonal gammopathies in the clinical laboratory. It has also replaced the classical immunoelectrophoresis because of its simplicity of interpretation. In addition, it is also an extremely sensitive procedure because of the combination of antigen and antibody, which increasing amount of dye uptake of almost 10-fold.

However, as with any immunologic method, attention to the Heidelberger equivalency curve of antigen or antibody excess must be considered when interpreting the procedure. Either antigen or antibody excess may render the precipitin band unreadable. This is especially true with Bence Jones proteins in urine IFE studies.

The literature provided evidences that immunofixaction technique sometime failed in identification of monoclonal components. Procognoli and his colleagues identified 6 serum samples from 6 patients in which immunofixation failed the identification of slight anomalous peak [15]. Yang et al. reported some exceptions about the usefulness of immunosubtraction in the identification of small IgA monoclonal components [16]. Immunosubtraction could detect low-abundant proteins in serum samples and in some cases it considered a more accurate method.

\section{Conclusion}

In the light of our data, we considered immunosubtraction method a third level test, particularly in that cases when immunofixation failed the identification of MC. Some techniques are considered "old and obsolete" but when the "new" failed the combination of "old and new" became the gold standard. Immunosubtraction takes a new life, the "day of future past" in limited cases.

\section{References}

[1] Dispenzieri, A., Katzmann, J.A., Kyle, R.A., et al. (2010) Prevalence and Risk of Progression of Light-Chain Monoclonal Gammopathy of Undetermined Significance: A Retrospective Population-Based Cohort Study. Lancet, 375, 1721-1728. https://doi.org/10.1016/S0140-6736(10)60482-5

[2] Kyle, R.A., Therneau, T.M., Rajkumar, S.V., et al. (2006) Prevalence of Monoclonal Gammopathy of Undetermined Significance. The New England Journal of Medicine, 354, 1362-1369. https://doi.org/10.1056/NEJMoa054494

[3] Landgren, O., Linet, M.S., McMaster, M.L., et al. (2006) Familial Characteristics of Autoimmune and Hematologic Disorders in 8,406 Multiple Myeloma Patients: A Population-Based Case-Control Study. International Journal of Cancer, 118, 30953098. https://doi.org/10.1002/ijc.21745

[4] Smith, A., Roman, E., Howell, D., et al. (2010) The Haematological Malignancy Research Network (HMRN): A New Information Strategy for Population Based Epidemiology and Health Service Research. British Journal of Haematology, 148, 739753. https://doi.org/10.1111/j.1365-2141.2009.08010.x

[5] Kyle, R.A. and Rajkumar, S.V. (2007) Epidemiology of the Plasma-Cell Disorders. 
Best Practice \& Research Clinical Haematology, 20, 637-664. https://doi.org/10.1016/j.beha.2007.08.001

[6] Varettoni, M., Corso, A., Cocito, F., et al. (2010) Changing Pattern of Presentation in Monoclonal Gammopathy of Undetermined Significance: A Single-Center Experience with 1400 Patients. Medicine, 89, 211-216. https://doi.org/10.1097/MD.0b013e3181e763be

[7] Kyle, R.A., Therneau, T.M., Rajkumar, S.V., et al. (2002) A Long-Term Study of Prognosis in Monoclonal Gammopathy of Undetermined Significance. The New England Journal of Medicine, 346, 564-569. https://doi.org/10.1056/NEJMoa01133202

[8] van de Donk, N.W., Palumbo, A., Johnsen, H.E., et al. (2014) The Clinical Relevance and Management of Monoclonal Gammopathy of Undetermined Significance and Related Disorders: Recommendations from the European Myeloma Network. Haematologica, 99, 984-996. https://doi.org/10.3324/haematol.2013.100552

[9] Kyle, R.A. and Rajkumar, S.V. (2007) Monoclonal Gammopathy of Undetermined Significance and Smoldering Multiple Myeloma. Hematology/oncology Clinics of North America, 21, 1093-1113. https://doi.org/10.1016/j.hoc.2007.08.005

[10] Munshi, N.C. (2007) Monoclonal Gammopathy of Undetermined Significance: Genetic vs Environmental Etiologies. Mayo Clinic Proceedings, 82, 1457-1459. https://doi.org/10.1016/S0025-6196(11)61086-0

[11] Blade, J. (2006) Clinical Practice. Monoclonal Gammopathy of Undetermined Significance. The New England Journal of Medicine, 355, 2765-2770. https://doi.org/10.1056/NEJMcp052790

[12] Blade, J., Rosinol, L., Cibeira, M.T., et al. (2008) Pathogenesis and Progression of Monoclonal Gammopathy of Undetermined Significance. Leukemia, 22, 1651-1657. https://doi.org/10.1038/leu.2008.203

[13] Kyle, R.A., Therneau, T.M., Rajkumar, S.V., et al. (2004) Long-Term Follow-Up of 241 Patients with Monoclonal Gammopathy of Undetermined Significance: The Original Mayo Clinic Series 25 Years Later. Mayo Clinic Proceedings, 79, 859-866. https://doi.org/10.4065/79.7.859

[14] Mollee, P. (2009) Current Trends in the Diagnosis, Therapy and Monitoring of the Monoclonal Gammopathies. The Clinical Biochemist Reviews, 30, 93-103.

[15] Pocognoli, A., Frezzotti, A., Galeazzi, M., et al. (2015) Immunosubtraction Technique Is Sometimes More Useful than Immunofixation for Typing IgA Monoclonal Components. Biochimica Clinica, 39, 326-330.

[16] Yang, Z., Harrison, K., Park, Y.A., et al. (2007) Performance of the Sebia CAPILLARYS 2 for Detection and Immunotyping of Serum Monoclonal Paraproteins. American Journal of Clinical Pathology, 128, 293-299. https://doi.org/10.1309/1L3CG8GK6F8VYNYH 